\title{
Production of carbonaceous materials with high capacitance by electrochemical tech- nique
}

\author{
Svetlana Kashina ${ }^{1}$, Marco Balleza ${ }^{2}$, Araceli Jacobo-Azuara ${ }^{1}$ and R. Galindo ${ }^{3, *}$
}

${ }^{1}$ Department of chemistry, Natural and Exact Sciences Division, University of Guanajuato. Noria Alta S/N, Guanajuato, Guanajuato, Mexico 36050 ${ }^{2}$ Department of physics engineering, Sciences and Engineering Division, University of Guanajuato. Loma del Bosque \#103, Lomas del Bosque, Lomas del Campestre, León, Guanajuato, Mexico, 37150

${ }^{3}$ CONACyT cathedra, Natural and Exact Sciences Division, University of Guanajuato

Corresponding Author Email: galindorosario@gmail.com

\begin{abstract}
Electrochemical supercapacitors are widely investigated as energy storage devices. The major part of research is dedicated to electrode materials development. Herein, we describe an electrochemical synthesis of 6 carbon-based materials from graphite. Influence of synthesis parameters (temperature and distance between electrodes) on resulting materials was investigated. The materials were tested to be used as electrode material in electrochemical energy storing system with phosphate buffer $(\mathrm{pH}=7)$ as supporting electrolyte. Resulted system showed a good stability and high operating voltage range $(0-1.1 \mathrm{~V})$. The higher and most stable specific capacitance of discharge was shown for a system there the material obtained at $80^{\circ} \mathrm{C}$ and $3 \mathrm{~cm}$ of distance between electrodes was used as an electrode material $\left(207 \mathrm{~F} \mathrm{~g}^{-1}\right.$ and $23 \%$ after $200 \mathrm{cycles}$, respectively).
\end{abstract}

Keywords: electrochemical synthesis, carbon material, electrochemical supercapacitor

Received: July-05-2018, Accepted: January-24-2019, https://doi.org/10.14447/jnmes.v22i2.a08

\section{INTRODUCTION}

"Green" energy production and storage is a rapidly growing area of research due to increased demand of society. Commonly used lithiumion and lead based batteries can reach energy densities up to $180 \mathrm{~W} \mathrm{~h}$ $\mathrm{kg}^{-1}$ or higher, but they present slow energy delivery/uptake. Electrochemical capacitors, on the other hand, deliver high power and can work stably through many cycles. Their properties make them promising option to fill the gap between electrostatic capacitors and batteries. Electrochemical capacitors can be divided in two groups by their action mechanism: 1) electrical double layer capacitors, in which energy is stored in electrical double-layer formed at a solid electrode - electrolyte interface, so there is no charge transfer $(1)$; 2) pseudocapacitors, there the energy is stored by fast reversible Faradaic reactions between the electrode and the ions in the electrolyte, so it involves charge transfer across the interface (2). It was shown that pseudocapacitors can deliver higher specific capacitance, so a lot of studies were dedicated to a search for appropriate electrode materials and working conditions. As mentioned above, supercapacitors store energy by ion adsorption, so specific surface area (SSA) of electrode materials must be relatively high to facilitate the process.

Carbon based materials are prominent in electrode development because of their unique properties. Many allotropic forms of carbon were studied to be used in supercapacitors. The most promising results were obtained for graphene and other shapes of carbon, due to their high surface area $(3,4)$. Graphene is considered one of the most promising materials for electrode development due to its electrical conductivity and a high theoretical specific surface area (up to $2630 \mathrm{~m}^{2} \mathrm{~g}^{-1}$ ) (5). Although, this area was not achieved in practice, $135 \mathrm{~F} \mathrm{~g}^{-1}$ gravimetric capacitance was reported for graphene in aqueous electrolytes (6). A gravimetric capacitance for graphene materials was improved $\left(298 \mathrm{~F} \mathrm{~g}^{-1}\right.$ ) in ionic liquids (3). Another way to improve gravimetric capacitance is doping graphene materials with nitrogen. For instance,
Lin et al. in 2015 reported synthesis of nitrogen doped few-layer carbon with $855 \mathrm{~F} \mathrm{~g}^{-1}$ in acidic conditions. Authors conclude that defects induced by nitrogen to graphene sheets convert them into active substance (7).

Carbon nanotubes (CNT) also were tasted for their application as electrode material for supercapacitors. It was shown that capacitances exhibited by CNTs can reach $180 \mathrm{~F} \mathrm{~g} \mathrm{~g}^{-1}$ in aqueous electrolytes and 70 $\mathrm{F} \mathrm{g}^{-1}$ in organic electrolytes, and it depends on structural parameters such as diameter and length $(8,9)$.

Relatively new micrometric form of carbon - porous carbon sphereswas tested recently to be used in electrochemical supercapacitors. Wickramaratne et al. in 2014 reported a production of nitrogen doped carbon spheres with SSA up to $1224 \mathrm{~m}^{2} \mathrm{~g}^{-1}$ and specific capacitance about $388 \mathrm{~F} \mathrm{~g}^{-1}$ in acidic conditions (10).

As mentioned above, these materials present high specific capacitance, but in order to achieve better capacitance values, acidic conditions or ionic liquids should be employed. Also, their synthesis requires essential use of specific chemical agents and equipment.

Electrochemical methods are used successfully to produce metal nanoparticles (11). In general, this method consists in application of constant current to anode, which begins to corrode giving place to nanoparticles. It was noticed that temperature of support electrolyte and the distance between electrodes are important parameters to control particles size. Where is not much information regarding to production of carbon materials using electrochemical systems. For instance, Wang in 2011 and Yin in the same year reported production of carbon nanospheres and graphene nanosheets using graphite rods as electrodes and $2 \mathrm{M} \mathrm{KNO}_{3}$ as a support electrolyte $(12,13)$. These materials were not tested for specific capacitance, but the "green" electrochemical synthesis may be a novel option to synthetize new carbon-based materials for electrochemical supercapacitors.

Considering the information described above, our research group 

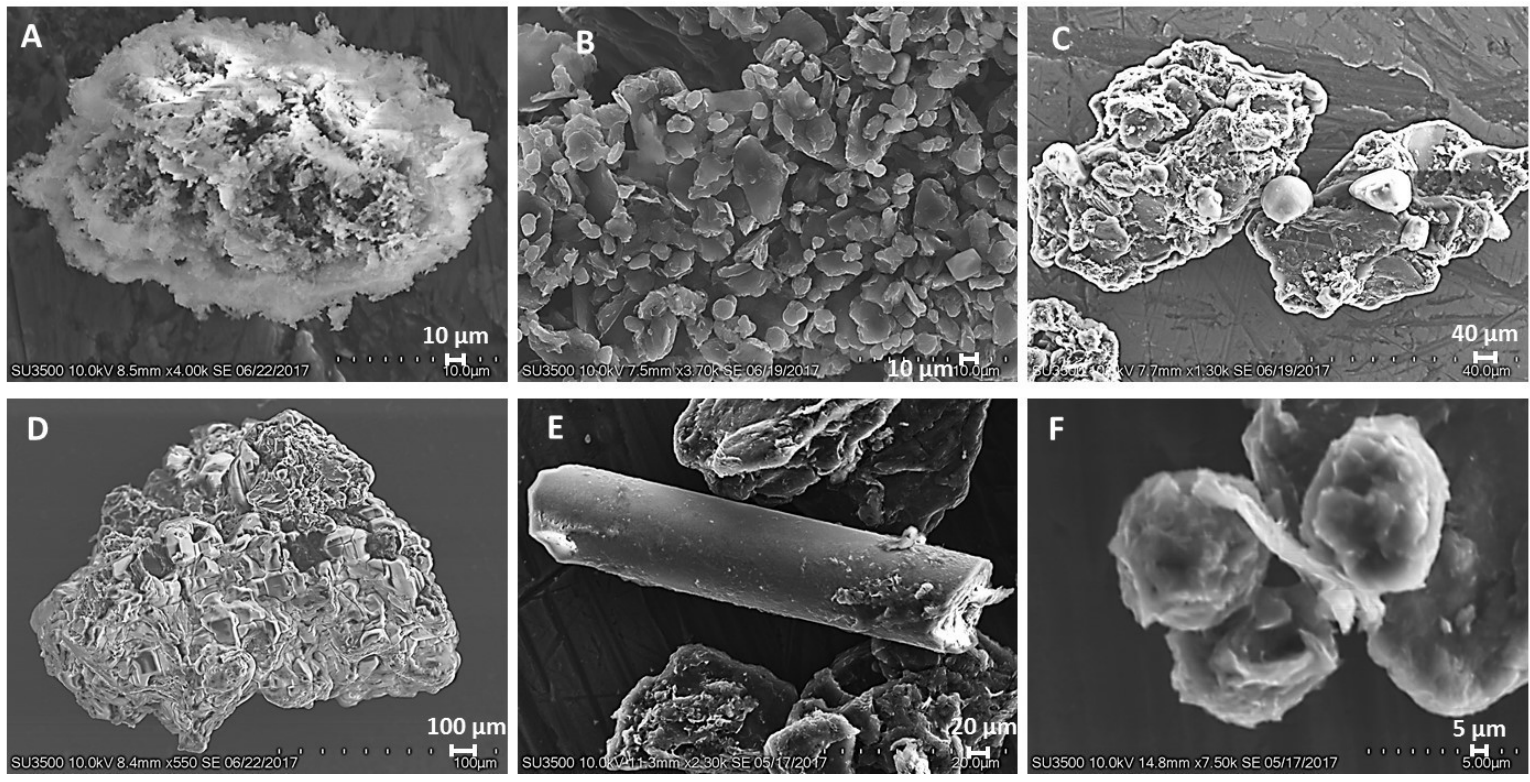

Fig. 1. SEM images obtained for materials: A) $\mathrm{KNO}_{3}-25-3$; B) $\mathrm{KNO}_{3}-50-3$; C) $\mathrm{KNO}_{3}-80-3$; D) $\mathrm{KNO}_{3}-80-1$; E) $\mathrm{KNO}_{3}-80-4$; F) $\mathrm{KNO}_{3}-80-5$.

decided to produce carbonaceous materials with relatively high capacitance by electrochemical technique using conventional graphite rod as starting material and test them as electrode material for electrochemical supercapacitors under neutral conditions.

\section{EXPERIMENTAL}

\subsection{Synthesis}

Two identical conventional graphite rods were used as electrodes. 2 $\mathrm{M}$ solution of $\mathrm{KNO}_{3}$ (KEM, Mexico) was prepared with distillated water. Electrodes were submerged into electrolyte solution and connected to the power source (PRL-25, Steren, Mexico). When a constant power of $5 \mathrm{~V}$ was applied to the system, anode began to corrode, and a black precipitate was formed. Experiments were conducted until a desired amount of precipitate was formed ( $2 \mathrm{~h}$, approximately). The precipitate was collected and washed with distillated water and ethanol and dried. Temperature of synthesis and distance between electrodes were varied: 25,50 and $80{ }^{\circ} \mathrm{C}$ and $1,3,4$ and $5 \mathrm{~cm}$, respectively. The materials were named as $\mathrm{KNO}_{3}-\mathrm{X}-\mathrm{Y}$, there $\mathrm{X}$ is temperature of synthesis and $\mathrm{Y}$ is a distance between electrodes.

\subsection{Characterization}

Scanning electron microscopy was performed by SU 3500 SEM (Hitachi, Japan). Specific surface area and pore size were determined using Tristar II plus (Micrometrics, USA). Raman spectroscopy was performed by DXR ${ }^{\mathrm{TM}}$ Raman Microscope (Thermo Fisher Scientific, USA). Low pass filter (cut frequency $0.01 \mathrm{~Hz}$ ) was applied to Raman signal in order to remove high frequency noise.

\subsection{Electrochemical characterization}

All electrochemical experiments were performed by VSP potentiostat system (Bio-Logic Science Instruments, France). Conventional three electrode cell was used for all electrochemical experiments. Inks were prepared form each material with isopropanol and Nafion ${ }^{\circledR}$ (Sigma Aldirch) and deposed by drop cast in a surface of glassy carbon electrode. A platinum foil was used as counter electrode and saturated calomel electrode (SCE) as reference. PBS $\left(\mathrm{pH}=7, T=25^{\circ} \mathrm{C}\right)$ was used as support electrolyte. Cyclic voltamograms were performed with scan rate $10 \mathrm{mV} \mathrm{s}^{-1}$. Electrochemical impedance spectroscopy was per- formed at sinus amplitude of $250 \mathrm{mV}$ and frequency range $5 \mathrm{mHz}-10$ $\mathrm{kHz}$. Galvanostatic charge-discharge experiments were made at $1 \mathrm{~A} \mathrm{~g} \mathrm{~g}^{-1}$ current density

\section{RESULTS AND DISCUSSION}

\subsection{SEM characterization}

A process of electrochemical decomposition of graphite was applied to produce novel materials. Considering that temperature is known factor that influence particle size during the synthesis and properties of resulted materials, we conducted electrochemical synthesis in three temperature conditions: 25,50 and $80{ }^{\circ} \mathrm{C}$. Figure 1 shows SEM images of 6 obtained materials. First three (A, B and C) correspond to materials synthetized at 25,50 and $80{ }^{\circ} \mathrm{C}$, respectively. It can be observed that shapes and size of the particles of materials varied without any clear tendency.

SEM images show that particles of all obtained materials are micrometric sized. The results did not present any correlation between temperature and particles size. This finding does not conclude with studies performed with metal nanoparticles, where particle size was reduced with temperature increase (11). The difference can be explained by the nature of studied materials. In our study, the smallest particle size was obtained at $50{ }^{\circ} \mathrm{C}$, and at $80^{\circ} \mathrm{C}$ a sphere-liked shapes started to form.

Distance between electrodes is another parameter to be attended during electrochemical synthesis $(14,15)$, so we used $1,3,4$ and $5 \mathrm{~cm}$ of distance between electrodes. Parts C-F of Figure 1 are images of materials obtained using 3,1,4 and $5 \mathrm{~cm}$ distance between electrodes, respectively. It can be observed that at $1 \mathrm{~cm}$ distance the material become smoother, but still have irregular shape, meanwhile, at lager distance defined shapes (spheres and rods) starts to appear.

It was shown that distance between electrodes have influence on particles shape and size. This effect can be explained by the fact that the larger is the distance between electrodes, greater will be the contribution of medium resistance, thus electrical current in the system will become smaller.

\subsection{Specific surface area}

As mentioned earlier, specific surface area is a key point in supercapacitors development, so this parameter was assessed for all synthe- 




Fig. 2. Raman spectra of materials: A) $\mathrm{KNO}_{3}-25-3$; B) $\mathrm{KNO}_{3}-50-3$; C) $\mathrm{KNO}_{3}-80-3$; D) $\mathrm{KNO}_{3}-80-1$; E) $\mathrm{KNO}_{3}-80-4$; F) $\mathrm{KNO}_{3}-80-5$.

tized materials. Table 1 comprise the calculated specific surface area (SSA) and pore size for each material. It was evidenced that SSA do not vary in great range between the materials, and the greater SSA was obtained for material $\mathrm{KNO}_{3}-80-1$. These results coincided with size and shape of particles obtained by SEM, thus materials with smaller particle size show lager surface area.

\subsection{Raman spectroscopy}

To assess the surface properties and crystallinity of the materials, Raman spectroscopy was used. Figure 2 shows Raman signal and Table 2 resumes obtained information. Two main peaks are distinguishable in spectra - 1314 and $1584 \mathrm{~cm}^{-1}$ (D and G bands, respectively). D band corresponds to the disturbances in $\mathrm{sp}^{2}$ configuration, meanwhile, $\mathrm{G}$ band corresponds to perfect $\mathrm{sp}^{2}(16)$. The ratio between the peaks (G/D) shows the grade of the disorder of carbon material. The ratios obtained for all synthetized materials were about $60 \%$.

So, characterization of materials surface by Raman confirmed carbonaceous nature of obtained materials. Taking to account the G/D ratios

Table 1. BET surface data

\begin{tabular}{ccc}
\hline Material & $\mathrm{BET}\left(\mathrm{m}^{2} / \mathrm{g}\right)$ & Diameter of pores $(\mathrm{nm})$ \\
\hline $\mathrm{KNO}_{3}-25-3$ & 2.9 & 5.8 \\
$\mathrm{KNO}_{3}-50-3$ & 6.7 & 5.7 \\
$\mathrm{KNO}_{3}-80-3$ & 3.2 & 5.8 \\
$\mathrm{KNO}_{3}-80-1$ & 3.9 & 5.9 \\
$\mathrm{KNO}_{3}-80-4$ & 4.2 & 5.8 \\
$\mathrm{KNO}_{3}-80-5$ & 2.3 & 5.9 \\
\hline
\end{tabular}

and the fact that in the spectra where is no apparent $\mathrm{G}^{\prime}$ band (typical for graphite), it may be concluded that electrochemically synthetized materials suffered structure modifications and are not parted graphite.

\subsection{Electrochemical characterization}

To evaluate electrochemical behavior and a working voltage window, a cyclic voltamperogramms were obtained in different voltage ranges (Figure 3). It can be observed that all materials except $\mathrm{KNO}_{3}$ 80-5 present a rectangular shape, typical for capacitors. Inside the voltage window $0-1.5 \mathrm{~V}$ all systems do not reach nor anodic nor cathodic barrier, but the capacitive behavior is present only in $0-1.1 \mathrm{~V}$ range, approximately. Reaching this potential is very important in electrochemical capacitors development, since the experiments were performed in neutral aqueous solution and can be compared to acid based ones. At the end of each voltage window, a current increase can be observed due to redox reactions which take place at the electrode (17).

Figure 4 presents Nyquist impedance plots with insertions of Bode plot corresponding to each material. From $4 \mathrm{~A}$, it can be observed that the behavior at medium to low frequencies of materials $\mathrm{KNO}_{3}-25-3$, $\mathrm{KNO}_{3}-50-3$ and $\mathrm{KNO}_{3}-80-3$ approaches a line parallel to Y-axis, typical for capacitors (18). The plot from 4B shows that the decrease of phase angle of capacitor was directly proportional to increase of temperature of synthesis of materials.

On the other hand, Nyquist plots obtained for materials $\mathrm{KNO}_{3}-80-1$, $\mathrm{KNO}_{3}-80-3, \mathrm{KNO}_{3}-80-4$ and $\mathrm{KNO}_{3}-80-5$ show that only materials obtained at 1,3 and $5 \mathrm{~cm}$ of distance between electrodes present capacitor-like behavior, meanwhile, Nyquist plot of material $\mathrm{KNO}_{3}-80-4$ at

Table 2. Raman spectra analysis

\begin{tabular}{|c|c|c|c|c|c|}
\hline \multirow{2}{*}{ Material } & \multicolumn{2}{|c|}{ Raman shift $\left(\mathrm{cm}^{-1}\right)$} & \multicolumn{2}{|c|}{ Peak area } & \multirow{2}{*}{ Ratio } \\
\hline & $\mathrm{D}$ & $\mathrm{G}$ & $\mathrm{D}$ & G & \\
\hline KNO3-25-3 & 1316 & 1585 & 18497 & 10936 & 0.59 \\
\hline KNO3-50-3 & 1314 & 1582 & 14167 & 8923 & 0.63 \\
\hline KNO3-80-3 & 1315 & 1586 & 15361 & 8697 & 0.57 \\
\hline KNO3-80-1 & 1316 & 1584 & 14419 & 8466 & 0.59 \\
\hline KNO3-80-4 & 1312 & 1583 & 15626 & 13240 & 0.85 \\
\hline KNO3-80-5 & 1313 & 1582 & 17931 & 10468 & 0.58 \\
\hline
\end{tabular}




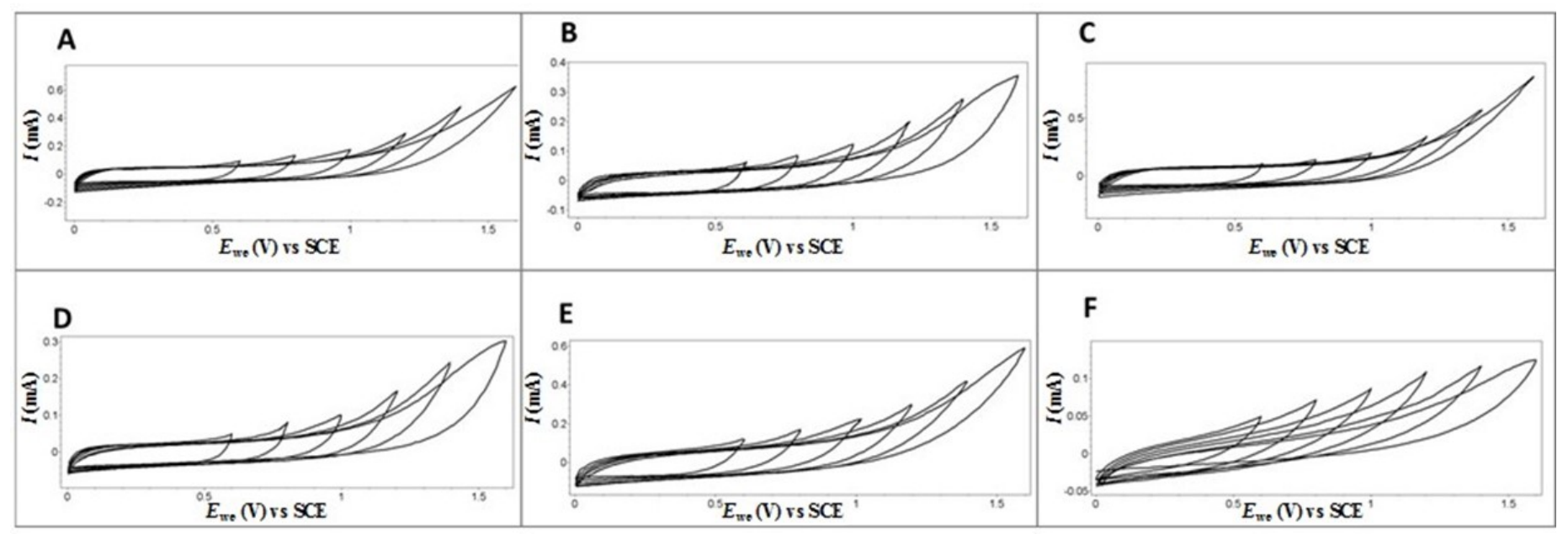

Fig. 3. Voltamperogramms obtained for materials: A) $\mathrm{KNO}_{3}-25-3$; B) $\mathrm{KNO}_{3}-50-3$; C) $\mathrm{KNO}_{3}-80-3$; D) $\mathrm{KNO}_{3}-80-1$; E) $\mathrm{KNO}_{3}-80-4$; F) $\mathrm{KNO}_{3}-80-5$. (Scan rate $10 \mathrm{mV} / \mathrm{s}$, vs $\mathrm{SCE}$ ).

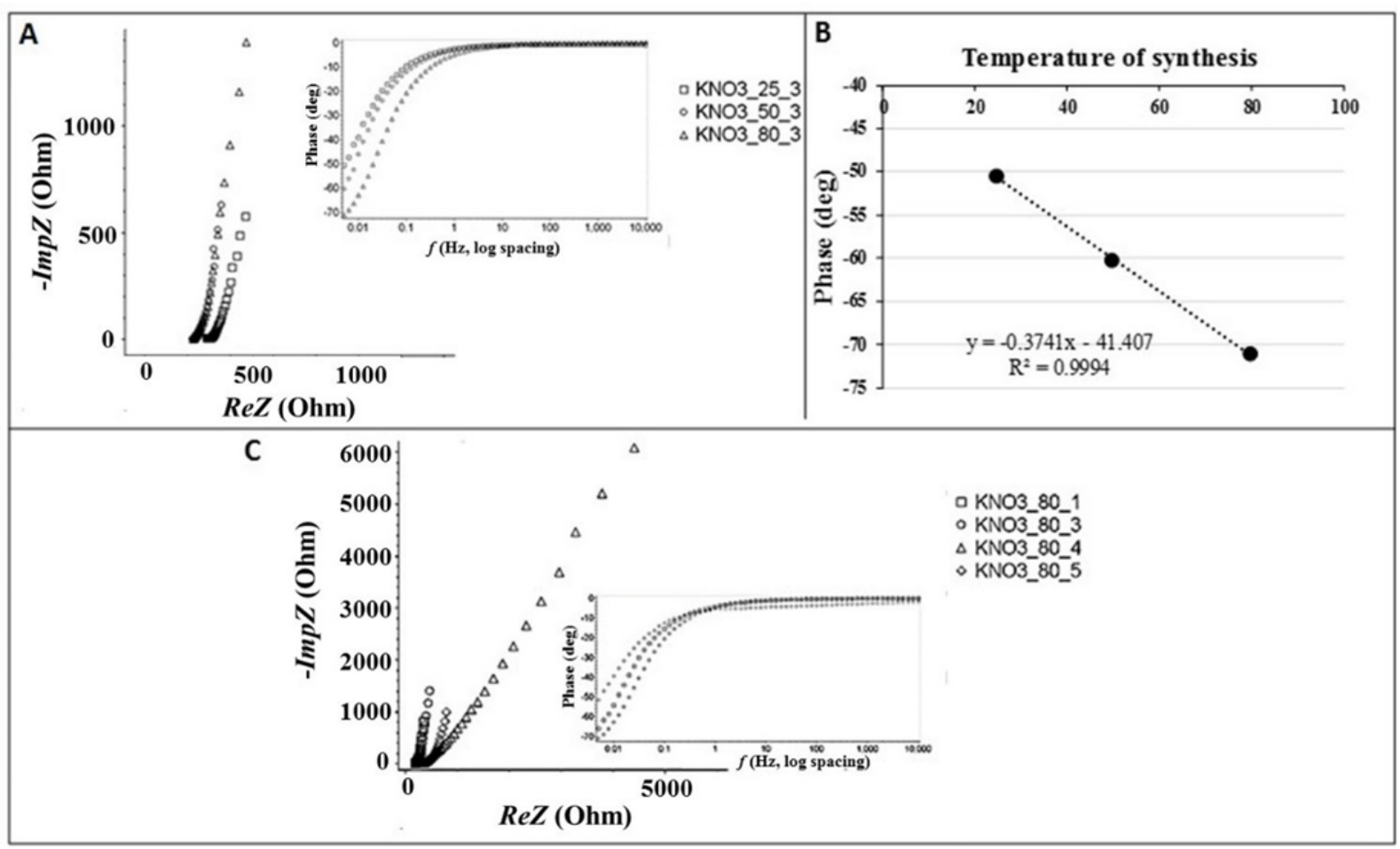

Fig. 4. Nyquist impedance. Insertions correspond to Bode plot for phase. A) Temperature dependence; B) Correlation between temperature of synthesis and phase angle; C) Dependence of distance between electrodes.

low frequencies approaches Warburg impedance, that model infinite diffusion process (19).

\subsection{Specific capacitance}

Specific capacitance measurement and its retention were assessed in a cyclic charge-discharge experiments (Figure 5). From the figure 5A it can be observed, that material synthetized at $25{ }^{\circ} \mathrm{C}$ presents greater specific capacitance at first cycle $\left(273 \mathrm{~F} \mathrm{~g} \mathrm{~g}^{-1}\right)$, but material $\mathrm{KNO}_{3}-80-3$ presents better retention after 200 cycles (23\% vs 34\%). Figure 5B shows that material $\mathrm{KNO}_{3}-80-5$ do not present any capacitance, the finding concludes with cyclic voltamperogramm obtained for this material. Material $\mathrm{KNO}_{3}-80-4$ presents some capacitance, but it decreases almost to zero during first 10 cycles. Capacitor formed with $\mathrm{KNO}_{3}-80$ 1 shows stability in discharge capacitance, but it is significantly lower than that obtained for $\mathrm{KNO}_{3}-80-3$.

Materials obtained in this study presented significant differences in capacitance and its retention. Possible explanation of this observation is capacity of materials to change the crystal structure according to temperature of synthesis. Probably, the synthesis temperature range was not enough to cause structure changes that can be observed by Raman spectroscopy, but was sufficient to provoke small changes that difficult current flow through material. As mentioned before, electrochemical supercapacitors store energy by adsorbing ions form electrolyte on surface of electrode. In order to do so, electrode materials must possess 


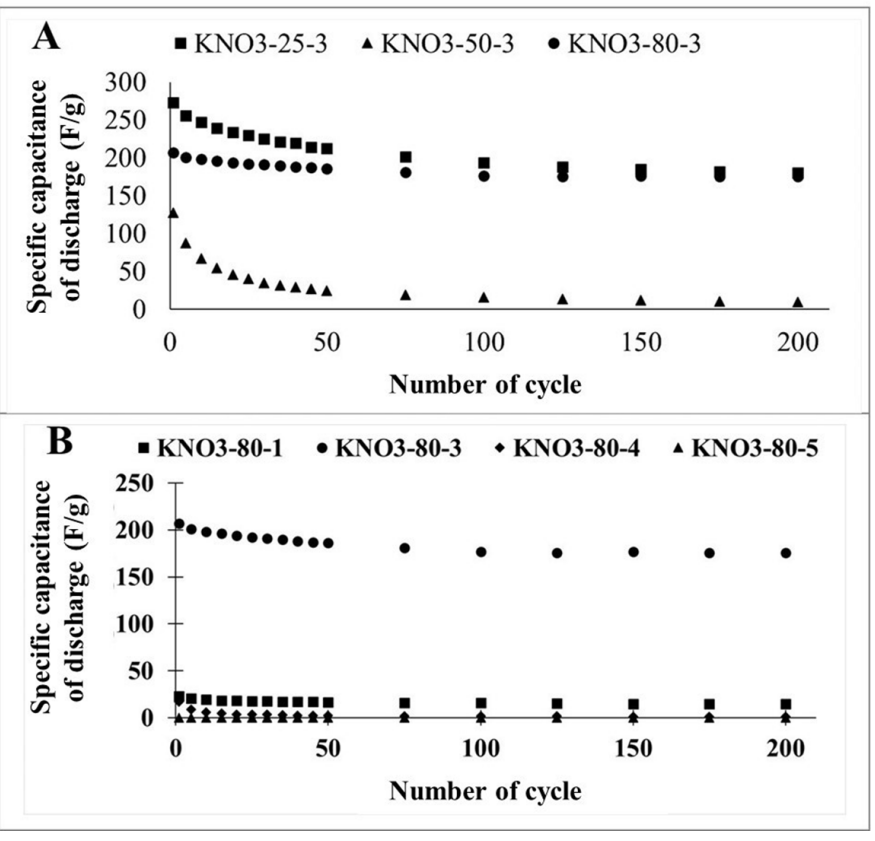

Fig. 5. Specific capacitance of materials. A) Temperature dependence; B) Dependence of distance between electrodes.

adsorption sites. Wang et al. in 2014 shown that adsorption capacity of graphene y graphene oxide towards polycyclic aromatic hydrocarbons was changed (20). Authors conclude that flexibility of sheets may be sufficient to cause conformational change of active sites. In present study, electrical current and conditions of synthesis (temperature and distance between electrodes) also could induce changes in adsorption sites, causing differences in capacitance behavior.

For authors knowledge, this study is a first attempt to use an easy electrochemical technique to produce carbonaceous materials that are suitable to by applied as electrode material in electrochemical supercapacitors

\section{CONCLUSIONS}

Six different carbonaceous materials were synthetized by electrochemical technique using graphite as starting material. Condition of synthesis were optimized to obtain materials with high specific capacitance and acceptable retention of capacitance during charge-discharge cycling. From the obtained results, it can be concluded that the material synthesized at $80{ }^{\circ} \mathrm{C}$ and at $3 \mathrm{~cm}$ distance between electrodes present the highest specific capacitance and it retention.

\section{ACKNOWLEDGEMENTS}

The autors thank CONACyT por scolarship for Svetlana Kashina (Number 442750).

\section{REFERENCES}

[1] Simon P, Gogotsi Y., Nat. Mater., 7(11), 845 (2008)

[2] Miller JR, Simon P., Science, 321(5889), 651 (2008).

[3] Xu YX, Lin ZY, Zhong X, Huang XQ, Weiss NO, Huang Y, et al., Nat. Commun., 5, 4554 (2014).

[4] Han J, Xu G, Ding B, Pan J, Dou H, MacFarlane DR., J. Mater. Chem. A, 2(15), 5352 (2014).

[5] Zhu YW, Murali S, Cai WW, Li XS, Suk JW, Potts JR, et al., Adv.
Mater., 22(46), 5226 (2010)

[6] Stoller MD, Park SJ, Zhu YW, An JH, Ruoff RS, Nano Lett., 8(10), 3498 (2008).

[7] Lin TQ, Chen IW, Liu FX, Yang CY, Bi H, Xu FF, et al., Science, 350(6267), 1508 (2015),

[8] Frackowiak E, Jurewicz K, Szostak K, Delpeux S, Beguin F., Fuel Process Technol., 77, 213 (2002).

[9] An KH, Kim WS, Park YS, Moon JM, Bae DJ, Lim SC, et al., Adv. Funct. Mater., 11(5), 387 (2001).

[10]Wickramaratne NP, Xu JT, Wang M, Zhu L, Dai LM, Jaroniec M., Chem. Mater., 26(9), 2820 (2014).

[11]Mazario E, Morales M, Galindo R, Herrasti P, Menendez N., J. Alloy Compd., 536, S222 (2012).

[12]Wang J, Yin HS, Meng XM, Zhu JY, Ai SY., J. Electroanal. Chem., 662(2), 317 (2011).।

[13]Yin H, Zhou Y, Meng X, Shang K, Ai S., Biosens Bioelectron, 30(1), 112 (2011).

[14]Cabrera L, Gutierrez S, Menendez N, Morales MP, Heffasti P., Electrochim Acta, 53(8), 3436 (2008).

[15]Lavorante MJ, Franco JI, Bonelli P, Imbrioscia GM, Fasoli HJ, J. Energy Power Sources, 1(3), 123 (2014).

[16]Ferrari AC, Basko DM., Nat. Nanotechnol, 8(4), 235 (2013).

[17]Demarconnay L, Raymundo-Pinero E, Beguin F., Electrochem. Commun., 12(10), 1275 (2010).

[18]Orazem ME, Tribollet B., Electrochemical impedance spectroscopy: John Wiley \& Sons, 2011.

[19]Lasia A., Electrochemical impedance spectroscopy and its applications: Springer, 2014.

[20]Wang J, Chen ZM, Chen BL., Adsorption of Polycyclic Aromatic Hydrocarbons by Graphene and Graphene Oxide Nanosheets. Environ Sci Technol 48(9):4817-25 (2014). 